\title{
Post-Disaster Mental Distress Relief: Health Promotion and Knowledge Exchange in Partnership with a Refugee Diaspora Community
}

\author{
Laura Simich, Lisa Andermann, Joanna Anneke Rummens, and Ted Lo
}

\begin{abstract}
After the 2004 Asian tsunami, a group of concerned scientists, physicians, and community service providers united to form a Local Distress Relief Network (LDRN) to provide information, referral, and care to affected members of the large Sri Lankan Tamil community in Toronto. The LDRN organized a workshop that brought together community-based organizations and international and local experts in mental health and disaster response to review existing knowledge on disaster response and to share community knowledge and experience. This article summarizes the development of the network, the workshop proceedings and joint recommendations for communitybased, culturally appropriate mental distress relief.
\end{abstract}

\section{Résumé}

Après le tsunami survenu en Asie en 2004, un groupe concerné de scientifiques, médecins, et fournisseurs de services communautaires se sont unis pour former un réseau local de soulagement de la détresse (Local Distress Relief Network - LDRN) dans le but de fournir des renseignements, de référer, et de prodiguer des soins aux membres affectés de l'importante communauté tamoule sri lankais à Toronto. Le LDRN a organisé un atelier de travail qui a rassemblé des organisations communautaires ainsi que des experts internationaux et locaux dans le domaine de la santé mentale et d'intervention en cas de désastres pour passer en revue les connaissances existantes en matière de réponses aux désastres et partager le sa- voir et l'expérience de la communauté. Cet article résume le développement du réseau, les délibérations de l'atelier de travail et les recommandations communes pour le soulagement de la détresse mentale adapté à la communauté et culturellement approprié.

\section{Introduction}

In response to the devastating effects of the December 26, 2004, tsunami in Asia, a group of concerned scientists, physicians, and community service providers came together in Toronto to form a Local Distress Relief Network (LDRN). The goal of the Network was to provide a locally targeted, responsive system of information, referral, and care to assist affected members of the Sri Lankan Tamil community in Toronto (population 200,000) ${ }^{1}$ - the largest Tamil diaspora in the world - which was deeply and directly affected by the disaster. This was not the first time the Tamil community had suffered. The Tamil population in Canada originated as refugees fleeing war and persecution in Sri Lanka, and thus had endured previous dislocation, psychosocial trauma, and resettlement stress. ${ }^{2}$ Even before the tsunami inundated the homeland, appropriate mental and other health services had not been available to meet Tamil community mental health needs. In a multicultural society such as Canada's, where numerous diaspora communities bind us all to world events, a vision for culturally appropriate and effective mental health services during and after emergencies was (and remains) critical. 
The ongoing controversy in mental health over trauma counseling and appropriate responses to disaster, whether focused on mental health treatment or social interventions, has produced principles for training ${ }^{3}$ and public health responses, ${ }^{4}$ which commonly and clearly express the importance of community-based interventions to aid recovery for affected populations. However, a "community" may be conceptualized as transnational, or defined by a "type of consciousness," thus having psychosocial as well as political and economic ties. ${ }^{5}$ Not only are survivors of natural disasters or conflict in resource-poor countries directly affected and at risk of mental distress, but also affected are members of those societies living in the diaspora in refugee resettlement countries, particularly families of those injured or killed. 6

To date, little attention has been devoted to understanding the mental health effects of far-flung diasasters or to devising an appropriate public and mental health response to support affected diaspora populations whose psychological ties to the homeland may be strong. Psychiatric epidemiology and studies of the effects of disasters have noted heightened vulnerability for women, children, ${ }^{7}$ and ethnic minorities with prior exposure to community violence and disparities in the availability and accessibility of mental health care. ${ }^{8}$ They have concluded that community-based interventions are most effective. Experts have also confirmed that psychosocial support interventions following disasters are best provided by understanding cultural context and how cultural factors can shape social responses and healing strategies. ${ }^{9}$

Our own participant observation in the LDRN initiative, begun as a way of addressing the psychosocial needs of the Sri Lankan Tamil community in Toronto after the tsunami, confirms that culturally appropriate and community-based distress relief can be successful when such initiatives support communities and unite the strengths of many actors. This article briefly describes the development the LDRN, and then describes the content of a joint workshop that was organized to exchange knowledge about distress relief models and practices that can promote recovery in both local and transnational contexts.

\section{Background}

Canada is one of the leading refugee resettlement countries in the world today, and Sri Lanka one of the world's leading refugee producing countries. Toronto is North America's most diverse city, with nearly half of its population born outside of Canada. Yet, despite a well-deserved reputation for multiculturalism and universal health care policies, Canada has not yet done enough to ensure that mental health care is accessible to its diverse newcomer populations. Needs for culturally appropriate mental health promotion, prevention, and care outstrip the available mental health services even in normal times. This is one reason that Toronto Tamil individuals and community organizations had approached Dr. Morton Beiser in the Culture, Community and Health Studies Program at the University of Toronto in 1999 to propose conducting a mental health survey in the Toronto Tamil community. The Community in Distress study was proposed, funded by the Canadian Institutes for Health Research, and carried out from 2000 to 2004 in partnership with several Tamil community groups. ${ }^{10}$

Social conditions in Canada, as well as pre-migration experiences, influence refugee community mental health. Some challenges facing the Tamil community are similar to those in other immigrant and refugee groups, and include experiences of displacement and exposure to traumatic events; limited comfort, especially among women, with using English; underemployment and discrimination in Canada; difficulties for seniors in terms of loss of freedom and social status; and lower than average household incomes during the early years in Canada. Nonetheless, Toronto Tamils form an increasingly well-educated community with a great deal of social capital. Most feel that they have good social relations and support among family and friends. Though nearly half of those interviewed in the study were forced to seek asylum in Canada, 65 per cent are now Canadian citizens. ${ }^{11}$ There is also a marked increase in prosperity after ten to fifteen years in Canada. This trajectory suggests that the Toronto Tamil community rises to challenges, as also is proven by the community's response to the tsunami.

The Local Distress Relief Network originated in the commitment of the Tamil study's research team to community collaboration and socially relevant research. When the tsunami struck, the team sought ways to translate this commitment into support for the Tamil disapora community of Toronto. While much of the public attention was focused on disaster relief efforts overseas, it was clear that there were also unmet needs for distress relief locally, for affected community members with worries or grief for loved ones back home, returnees from the region, and emergency disaster workers. The goal of the network became linking people and resources to facilitate knowledge exchange.

LDRN activities included information and networking meetings; preparation of a culturally competent flyer in Tamil that outlined common symptoms of distress and ways to address them and provided contact information for Tamil-speaking mental health care workers; distribution of this flyer at a public vigil for tsunami victims and via the Internet; preparation of an information letter concerning the impact of disasters on children and families for teachers 
and parents to be distributed in the school system; translation of a photo-novella on post-traumatic stress disorder into Tamil; and establishing a Tamil Mental Health Resource Group to promote and sustain referrals.

The LDRN succeeded because it was based on an established working relationship between researchers and community members that was rooted in trust and mutual respect. This made it possible to identify pressing needs and to focus on feasible tasks. It was important to let those affected within the community tell those willing to assist what they felt was most needed, and to support the community's own initiatives as much as possible. It was also important to share responsibilities to ensure efficiency, and to ensure response capacity before proceeding in order to meet heightened expectations. Although the network was started by a handful of colleagues, it belonged to everyone. The network thus strove to become a "coalition of leaders." Individual efforts and expertise contributed to a whole that was greater than the sum of its parts.

The LDRN was only a small part of the Toronto Tamil community's overall response to the tsunami, which preceded and extended well beyond the reach of the LDRN. As just one example, the Tamil Emergency Medical Services (TEMS), a registered non-profit, non-governmental organization based in Canada whose mandate is to enrich the lives of people affected by war through rehabilitation and development, filled pressing medical needs in the north and east of Sri Lanka by bringing together healthcare professionals, students, and community leaders. TEMS's tsunami-related projects included a locally organized medical supplies relief effort, a baby items drive, and support for Ponambalam Hospital and for health workers' training. In addition, members of the Tamil Service Providers' Coalition in Toronto provided support services to families after the tsunami and were also trained by Toronto Public Health to provide grief counselling. Tamil media organizations assisted by fundraising for immediate relief efforts and providing opportunities for individuals to talk about their suffering and comfort each other through call-in talk shows. Tamil places of worship also organized special prayer services throughout Toronto. To offset the fact that international disaster aid was not always delivered to the areas most in need, Tamil health professionals and other community members visited disaster-affected areas in the homeland to deliver medical, material, and emotional support directly. This massive community-led aid effort likely helped to assuage feelings of helplessness and loss and promoted rehabilitation and mental health in the larger community.
Organization of the Workshop "Mental Health in Public Health Policy and Practice: Providing Culturally Appropriate Services in Acute and Post-emergency Situations"

The desire to provide distress relief after the tsunami raised questions such as these: What should be the role of trauma counselling in good mental health practice after emergencies? What does this approach entail for diaspora and ethnocultural communities in Canada? What are the implications for public health, hospital, and community mental health practice in acute and post-emergency phases?

To address these issues, the authors organized a one-day workshop that brought together international, national, and local experts in health and disaster relief and community-based organizations to review existing literature, share practical experience and community knowledge, and discuss the implications for distress relief initiatives for affected ethnocultural populations. ${ }^{12}$ The workshop, entitled Mental Health in Public Health Policy and Practice: Providing Culturally Appropriate Services in Acute and Postemergency Situations, was designed to build on existing international and local expertise and to explore implications for Canadian public health policy and practice. Key tasks were to: (1) consider existing mental distress relief models and culturally appropriate public health practices, (2) explore implications for Canadian health research, policy, and service delivery, and (3) develop initial guidelines through consensus.

Held on May 26, 2005, at the Centre for Addiction and Mental Health in Toronto, the workshop benefited from the combined expertise and energy of participants from several sectors, including academic, community mental health care, and policy. Of the twenty-nine participants, four were mental health experts invited from outside of Canada. Local expert participants were drawn from Tamil and non-Tamil community health services, hospitals, and settlement service agencies, as well as municipal, provincial, and federal government agencies. The morning workshop program was devoted to presentations of distress relief models. The ensuing group discussions also incorporated "front-line" reports of local lessons and best practices by all workshop participants. The goal was to provide participants with an opportunity to exchange information, experience and ideas. In the afternoon, small working groups aimed at developing policy, practice, and research recommendations. This was followed by a full group discussion that pooled the recommendations, which were subsequently circulated to all participants to ensure consensus. Relevant background readings, summarized below, were distributed in advance of the workshop to help frame the planned group discussion. 


\section{Summary of Workshop Readings on Culturally Appropriate Models of Mental Distress Relief}

The tsunami of December 26, 2004, dealt a heavy blow to the already overburdened Tamil population in Toronto. The Community in Distress study completed just prior to the tsunami was the first survey of psychiatric epidemiology in this community and provided much needed background into the mental health needs of this population. ${ }^{13}$ Survey findings showed that approximately one-third of 1,600 Tamil respondents had experienced traumatic events including assault, rape, or witnessing combat. Of those who had experienced trauma, 36.2 per cent met criteria for posttraumatic stress disorder (PTSD), and the overall prevalence in the community was 12 per cent. Less than 10 per cent had received any specific psychological treatment. While 70 per cent of people had seen a general practitioner for various reasons, less than 1 per cent had seen a mental health worker or psychiatrist. This "poor fit" is observed between mental health needs and services and is associated with many barriers encountered including language, lack of information, stigma, and preferences for traditional healing.

The World Health Organization (WHO) statement "Mental and social health during and after acute emergencies: Emerging consensus?" summarized the opinions of world experts in a field which has been controversial. ${ }^{14}$ The authors provide a useful table of social and mental health intervention strategies and a discussion about emergency responses in a stepwise and multilevel manner. They describe eight basic principles of mental health in emergencies: (1) contingency planning, (2) assessment, (3) long-term perspective, (4) collaboration, (5) integration into primary health care, (6) access to service for all, (7) thorough training and supervision, and (8) monitoring. Much of the workshop discussion was based on these principles, with added emphasis on the diaspora situation.

In the Round Table discussion by Silove ${ }^{15}$ and Summerfield ${ }^{16}$ following the WHO consensus statement, Silove argues that "the best immediate therapy for acute stress is social," and that this would include providing safety, family reunification, effective justice systems, returning to productive roles in work or study, and "re-establishing systems of meaning and cohesion - religious, political, social and cultural." 17 When psychiatric disorders do occur and help is needed, he supports the use of community mental health programs integrated within primary care; however, he cautions that in resource-poor situations, some external resources and specialized training may be needed. Summerfield critiques the use of the PTSD concept in non-western populations, and is concerned that "psychosocial programs" imported to treat trauma in developing countries have tended to focus more on the psychological than the social. ${ }^{18}$ In fact, he believes this to be a false distinction of western thought promoting the dichotomy between mind and body, which is foreign in many other parts of the world. He writes:

Lack of coherence is bad for people: if there is such a thing as a core fact about human response to disasters and violent upheavals, it is that survivors do well (or not) in relation to their capacity to re-establish social networks and a viable way of life. Western mental health models have always paid too little attention on the role of social agency, including work, in promoting stable well-being and mental health. ${ }^{19}$

A survey of international experts conducted by Weiss and colleagues was also reviewed. ${ }^{20}$ Issues addressed include the nature and diversity of disasters, the validity of the PTSD diagnosis and the classification of traumatic stress responses, and goals and approaches for mental health policy and future directions for research. Of relevance to the tsunami, general opinion held that it was less important to differentiate between man-made and natural disasters than to develop different approaches for single event versus enduring trauma. Addressing sociocultural and community context was primary, including culturally sensitive needs assessment, using local expertise and recruiting local relief workers, and sustaining interventions over time. In addition, clinical and community interventions must be balanced, with mental health services being incorporated into primary health care. Development of stand-alone trauma services is not advised. Specifically, "aid programs should refrain from applying blanket trauma-focused clinical interventions to a large population, solely because the community as a whole has been exposed to disaster." 21

Training issues were thoroughly addressed by the consensus guidelines developed by the Task Force on International Training of the International Society for Traumatic Stress Studies (ISTSS). ${ }^{22}$ These guidelines focus on four main issues: (1) values underlying international training; (2) contextual challenges in societies during or after conflicts; (3) core curricular elements of training; and (4) monitoring and evaluation. Training must be culturally sensitive, as well as feasible and sustainable in the local setting, with some access to supervision. Self-care of mental health workers must be addressed. In addition, attention needs to be paid to monitoring, evaluation, and outcomes.

Although there is much written about cultural understanding of trauma and depression, one study by Eisenbruch on "cultural bereavement" in Southeast Asian refugees in the United States seemed particularly relevant for workshop participants in understanding possible re- 
sponses to the tsunami among Toronto Tamils, many of whom have already had direct experiences with trauma prior to coming to Canada. ${ }^{23}$ Cultural bereavement is described as "the experience of the uprooted person- or group-resulting from loss of social structures, cultural values and self identity... not of itself a disease, but an understandable response to the catastrophic loss of social structure and culture." ${ }^{24}$ Using semi-structured interviews and clinical vignettes to conduct an in-depth study of this syndrome, Eisenbruch suggested that disabling psychological symptoms may mimic post-traumatic stress disorder, but in fact will not respond to biomedical treatments or western psychotherapy alone. Cultural bereavement is an issue which goes beyond the symptoms of an individual. He adopts a wider perspective by asking:

If some of the people in this community come to a mental health resource with complaints such as sleeplessness or flashbacks or some other hallmarks of post-traumatic stress disorder, how are we really to treat them? Treating the symptoms can be counterproductive, because the patient returns to a community that is in itself in a state of collective grief and the patient becomes more estranged from the parent culture. ${ }^{25}$

He provides an answer emphasizing cultural meaning to achieve a truer understanding of the sufferer's experience, rather than symptoms to be medicated.

Furthering knowledge of community approaches, the work of Jack Saul describes strengths-based approaches to trauma that are grounded in the study of resilience. ${ }^{26}$ Saul conducted interviews with traumatized elderly Albanian Kosovars as "solution-oriented conversations" rather than standard psychological assessments in order to decrease their isolation and increase a sense of hope and problem solving. He writes, "by focusing on an individual's strengths and solutions, his or her problems are not denied but rather set in an atmosphere in which the individual can examine problems from a perspective of enhanced dignity and a sense of agency." $27 \mathrm{He}$ also addresses the importance of community structure, acknowledging that "many of the strengths that enable people to cope with massive loss and trauma are embedded in a community's collective memory, culture and religions," and that "when drawing on these resources, the community is able to tap the symbols, rituals and maps that have traditionally enabled it to navigate through very difficult like events and transitions." ${ }^{28} \mathrm{Cul}-$ tures each have their own "repertoire of healing mechanisms" and reconnecting people to these hidden or forgotten routes will enable them to overcome their traumatic experiences.
Landau and Saul describe working with a community resilience model in post-9/11 New York and Buenos Aires, Argentina, following a period of political and economic instability. ${ }^{29}$ The family is seen as the basic unit of "community," but the focus of intervention is broadened to the wider group. They identify four themes for community recovery, including: (1) social connectedness as foundation for recovery, (2) collective storytelling, (3) re-establishing daily routines and collective healing rituals, and (4) developing positive visions for the future. Community mapping is described as a first step in problem solving, and this brings the focus away from interventions on an individual level. Community members are approached as "natural change agents," allowing the success of the project to belong to the community.

Another contribution to existing knowledge comes from VIVO (Victim's Voice), an NGO that has been developing a short-term psychological intervention for post-traumatic stress disorder called Narrative Exposure Therapy (NET), ${ }^{30}$ which has also been researched in children. ${ }^{31}$ While mindful of the importance of community approaches, their expertise is in the area of treating individuals who are disabled by post-traumatic stress disorder and therefore unable to function. While other brief treatments for PTSD exist, NET is unique in that it has been shown to be effective in postconflict environments such as Uganda and Sri Lanka, can be done in the field with minimal resources, and most importantly, builds local competency through the use of trained laypersons. NET is taught to selected community members, such as the residents of a refugee camp, in a short period of time. They in turn treat others, with ongoing supervision and evaluation. Outcomes have been comparable to treatment by experienced NET therapists. This transition from "survivor" to "agent of change" and community healer is of great importance for local capacity building.

Workshop participants also reviewed a collection of first-hand reports and documents related to health and emergency measures and post-tsunami reconstruction in Sri Lanka, ${ }^{32}$ which emphasized listening to the concerns and preferences of the affected and reminded the international community of their responsibility to follow through on their commitments for assistance.

\section{Workshop Presentations: Expert Commentary on Existing Models of Distress Relief}

With the readings as shared background, guest speakers opened the workshop by presenting commentary and examples of good distress relief practices. Dr. Jose Bertolote, representing the WHO in Geneva, placed the day's issues in a global context. He noted that WHO received "unprece- 
dented" requests for mental health assistance following the tsunami. Making mental health a public priority would not have happened a mere five years before. Nevertheless, he noted that most emergency mental health responses remain ill-prepared. In the organization's experience, the most important lesson is that local realities necessarily shape local and external interventions. He advised against promoting new mental health services that are external to existing health services in emergency situations. However, he recommended promoting social interventions that are outside conventional mental health services. He supported offering basic psychological support, or what we have called "distress relief," both inside and outside the existing health system.

Bertolote acknowledged that the selective focus on PTSD in emergency mental health has been problematic, as has been the vertical organization of trauma services, the tendency to "parachute in" foreign trainers and clinicians and attempting to make a distinction between "mental health" and "psychosocial" services. Another problem has been the lack of baseline data on the impact of emergencies on mental health, although some assumptions can be made based on world surveys and disaster studies. Highly recommended responses at national and subnational levels include developing community-based mental health services, integrating these services into primary health care, and training personnel in all sectors to identify mental health issues and make appropriate referrals. He further emphasized that, unless cultural understanding is reached beforehand, emergency responses will only exacerbate the impact of the disaster.

Dr. Daya Somasundaram, Department of Psychiatry, University of Jaffna, Sri Lanka, contributed his ideas on community-level interventions, which are grounded in years of first-hand professional practice in the affected areas. He noted that most mental distress does not emerge as mental illness per se. Because mental distress does not arise as a medical problem, it should not be treated in a medical setting, but rather in the community. He described the effects of the December 2004 tsunami at several levels of society in northern Sri Lanka. At the individual and family levels, distressing experiences included loss of cohesiveness and unity; deaths, which created "vacuums"; family separations; disturbances in family dynamics; and family role change due to deaths of females. At the community level, experiences included widespread destruction of "organic roots" and breaks in the biological connections to the home; loss of support systems, networks, traditions, structures, and institutions; and loss of communality. He also described "collective trauma," which was characterized by an increased sense of dependency; deterioration in morals and values; poor leadership; adoption of superficial and short-term goals; prevalent mistrust and suspicion; and social deterioration exemplified by general resignation, loss of work ethic, and increasing alcoholism.

Somasundaram suggested that the goal of responses should be psychosocial well-being as defined by the WHO, that is, health as "a state of complete physical, mental, (familial), social and (spiritual) well-being, and not merely an absence of disease or infirmity." He recommended several effective therapeutic interventions for disaster survivors, including crisis intervention, psychotherapy, behavioural-cognitive methods, pharmacotherapy, relaxation techniques (western principles and traditional practices), massage, and group therapy (small groups with similar problems and support groups for caretakers). He stated that family therapy could work to promote unity, cohesiveness, and sharing of burdens and responsibilities; increase mutual understanding, communications and interactions; fulfill roles and the need for respect; and create positive family dynamics and extended family support. He also stressed the utility of expressive methods, such as art, drama, writing and storytelling, and therapeutic play, which promote individual and social healing, recovery, and reintegration.

Somasundaram emphasized the primacy of respecting the local population's wishes and needs and their active participation in useful tasks such as vocational training, agro-therapy, non-partisan cultural healing, and symbolic expression in media and public mourning. Linked with these family and community-based interventions, he observed, should be a referral system for mental health care and treatment. He highlighted the significance of indigenous coping strategies, including rituals and ceremonies such as funerals and festivals, and described the important role of traditional healers. Traditional methods are based on disaster survivors' cultural and religious beliefs, and are therefore less stigmatizing, more culturally acceptable and popular.

Four additional speakers addressed the workshop: two international experts, whose work was described in the literature reviewed, and two contributors from Toronto. Patience Onyut, a psychologist at the University of Konstanz, Germany, Refugee Outpatient Clinic, works with the University of Colombo, Sri Lanka, and VIVO, which treats patients and victims of violence and disaster who do not get better on their own. She presented applied research among children in northern and northeastern Sri Lanka, which demonstrates the potentially traumatic experiences of Tamil children from war, witnessing unexpected or violent death, threat with a weapon, witness of torture, harassment by armed personnel, and sexual abuse, with consequences of PTSD including somatic symptoms, suicidality, and im- 
paired school performance and memory. Onyut also described a second epidemiological study of children in Sri Lanka in January 2005, which showed a relatively high prevalence of PTSD three weeks after the tsunami. Predictors of PTSD included war experiences, domestic violence, and the severity of the tsunami itself, with a cumulative effect. Most relevant to culturally appropriate distress relief practices, Onyut described the successful use of the Cascade Model in which over 120 master counsellors supervise counsellors in Tamil schools, as well as 1,000 "befrienders" in all Tamil districts. The counsellors provide Narrative Exposure Therapy that has been shown to be effective for children using techniques such as drawing lifelines and other representations, acting, and body awareness.

Jack Saul, director of the International Trauma Studies Program, Columbia University, New York, spoke about facilitating community resilience following war and terrorism. Saul described his ten years of work with torture survivors in refugee communities and in post-9/11 downtown Manhattan. ${ }^{33}$ Saul defined resilience as "the capacity to rebound from adversity, strengthened and more resourceful. It is an active process of endurance, self-righting, and growth in response to crisis and challenge." The principles of the community resilience approach include the process of invitation; attaining authority, permission, and commitment from the community; the engagement of the entire community, including subgroups and all economic strata, the natural helping system; identification of scripts and themes across generations and community history; helping the community to map out its resources; turning goals into realistic tasks, and those tasks into practical projects; encouraging community members (natural "change agents") to become leaders; and finally, attributing success to the community.

Raymond Chung, executive director of Hong Fook Mental Health Association, Toronto, presented the Association's model of culturally appropriate care. Hong Fook is a community-based mental health organization which advocates "equal access" to achieve "mental health for all." Hong Fook has a notable history offering culturally appropriate mental health services in Toronto. The Association began early in the 1980s helping refugees and immigrants from war-torn Vietnam, and later Cambodia, adopting a Consultation Liaison Model by training bilingual/bicultural mental health workers to work with mainstream treatment facilities. Today, Hong Fook has forty-seven staff members working with newcomers from Cambodia, China, Hong Kong, Korea, Taiwan, and Vietnam. The guiding principles focus on empowerment, self help, mutual support, and capacity building. Chung outlined principles and assumptions used in working with distressed individuals and com- munities: migration is understood to be a stressful experience; migration and mental health are linked in the framework of social determinants of health; stigma attached to mental illness is a barrier to be overcome before any form of treatment or supportive strategies can be effective; and each individual and each diaspora or ethnoracial community is unique, yet continually changing. Service providers, Chung stated, have to "walk alongside the individuals and grow with the communities" to discover the strengths within each. Furthermore, he noted that prevention is better than treatment and that there are many alternative treatments to consider.

Dr. Clare Pain, director, Mount Sinai Hospital Psychological Trauma Assessment Clinic, Toronto, summarized the key themes. She pointed to a model of culturally appropriate distress relief that emphasizes the quality of relationships, specifically respectful, collaborative relationships among health care practitioners, service providers, policy makers, and communities. Pain observed that the most effective distress relief depends upon pre-existing relationships and knowledge. All too often a disaster or crisis occurs, chaos ensues, and the question only then arises, what interventions are appropriate? She noted that the wish to help confers strength, but also does possible harm. To avoid doing harm, it is important not to rush in to the situation, but to listen to requests first, then identify needs. She acknowledged that there are known vulnerable groups: children, elderly, and psychiatric patients in particular. Most people caught in crisis, however, are ordinary people, not patients (though some may not be able to function well for a while following a crisis). Pain also suggested that an underlying theme was the notion of the collective experience of distress, which is not necessarily caused by a single traumatic "event," but rather ongoing and extraordinary disruptions of normal life. Therefore, a reorientation in thinking is needed: going beyond the contemporary focus on PTSD, an individual disorder, to an analysis of collective trauma and ways to promote community healing. This new orientation might be thought of as a "bio-psychosocialcultural orientation."

\section{Perspectives from the Front Lines in Toronto: Workshop Participants' Experiences}

In the ensuing discussion by workshop participants, several specific challenges of providing distress relief were highlighted. Most service providers, who had first-hand experience dealing with the tsunami-affected Tamil population in Toronto, observed that the tsunami disaster had brought to the surface many long-standing problems and stresses associated with migration and settlement in Canada that had accumulated through the years. A few noted that disaster aid 
efforts also brought out feelings of anger and disappointment because of the inadequacies of the political response and inability of the Canadian government to follow through on promises made to deliver aid to tsunami-affected areas. Another source of frustration was misinformation about the government's intentions to fast-track affected family members through the immigration process. More positively, service providers noted that the community-based supportive programs that they were able to offer, particularly those in group settings that offered time and space for sharing emotional responses among clients and community members, appeared to be effective in alleviating mental distress for many people.

At an organizational level, workshop participants noted that the tsunami disaster response revealed problems such as jurisdictional barriers; the need for prior networking among agencies, professionals, and community workers; and the lack of response from mainstream institutions where mental health services are normally delivered. They observed that many institutions were unprepared to deal with disaster response and were uncertain about how to proceed to deal with intercultural communication. For example, in Toronto's schools, which were central gathering points for disaster-affected families and communities, people were generally not found to be receptive to available counselling literature about ways of dealing with psychosocial trauma. Furthermore, the political "hype" surrounding the tsunami disaster relief that motivated some institutional and government responses created skepticism in the end, because in immediately planning for "worst case" scenarios, some institutions and agencies requested or allocated resources for needs that did not materialize. Raising expectations thus hurt community members and may inhibit effective institutional responses in the future. Participants reiterated the importance of working with community organizations, securing commitments from mainstream institutions, and recognizing that linkages among families and the homeland may remain strong.

\section{Joint Recommendations for Culturally Competent Mental Distress Relief}

Based on their personal expertise and working group discussions, workshop participants developed recommendations for the provision of culturally appropriate policy, service provision, and research on distress relief. The recommendations are summarized below according to eight principles taken from background readings and two principles - Relations with other cultural communities and society, and Transnational ties - added to better acknowledge diaspora community experiences. Brief explanatory statements para- phrased from the WHO consensus document ${ }^{34}$ precede the recommendations.

\section{Contingency Planning and Assessment}

Before an emergency, national-level contingency planning should include interagency coordination, designing mental health response plans, and training general health care personnel in general mental health care and psychological first aid. Assessment should cover the sociocultural context (setting, culture, history, perceptions of illness, ways of coping), available services, resources, and needs.

- Identify different, relevant agencies and their scope of practice in advance.

- Involve communities in planning and assessment to ensure cultural/linguistic competence.

- Increase interaction between agencies and levels of government, horizontally and vertically.

- Determine who does what, and use this to determine responsibilities during disaster response.

- Develop clear lines of communication so that everyone is informed.

- Consult with and learn from the experiences of others. Identify and work with ethnocultural communities to learn from past experiences of dealing with trauma and disasters.

- Focus on needs identified by communities when developing a contingency plan, and on what is feasible in the current environment.

- Undertake distress relief initiatives in partnership with community leaders and organizations currently serving the community.

- Ensure that focus of action remains on community capacity building.

- Ensure ongoing review of contingency planning.

- Assess individuals in terms of ability or inability to perform daily functions.

- Consider the help-seeking behaviors of communities and impact on service delivery.

\section{Long-term Perspective and Collaboration}

Affected populations are helped most by a focus on mediumand long-term development of mental health services, although the needs are highest during or immediately after acute emergencies. Strong collaboration among agencies avoids waste and duplication. Continuous involvement of government, universities, and community agencies is essential for sustainability.

- Develop solutions that are long-term and sustainable.

- Ensure that policies and legislation (e.g. refugee legislation) are responsive to community needs, particularly during acute emergencies. 
- Be aware of the "social disconnectedness" experienced by refugee communities and seek to counter this during times of need.

- Engage in community and institutional capacity building (i.e. in hospitals and government).

- Facilitate collaboration among mental health organizations under the "public health" umbrella.

- Ensure that collaboration among service providers, health professionals, policy makers, community members, and researchers is mutually beneficial.

- Some communities may be hesitant to collaborate due to negative past experiences. Elicit the support of other communities in order to reach out to these communities.

- Remember that different partnerships (research, service provision, policy development) at different levels (local, intergovernmental, international) each have important roles to play.

- Ensure clarity with regards to roles, responsibilities, expectations, and contributions.

- Specifically charge individuals with the responsibility of coordinating collaborations.

Integration into Primary Healthcare and Access to Service for All

To ensure access to non-stigmatizing services for the largest number of people, mental health services should be made available within the primary health care sector. Setting up separate, vertical mental health care services is discouraged. At the same time, outreach is crucial to ensure treatment of vulnerable groups within general health and social services.

- Build services directly into existing structures to promote sustainability, increase the efficiency of the services, and better utilize resources.

- Shift away from compartmentalized care to a decentralized process that integrates mental health care into primary health care.

- Move distress-relief services from a health practitioner-centred model to a multidisciplinary model involving multiple sectors (e.g. schools, drop-in centres).

- Focus services on health promotion/prevention rather than treatment/reaction.

- Use a community-based approach to address mental health issues to reach the larger population and address collective trauma at the social level.

- Find ways to strengthen existing organizations that provide distress relief (e.g. the Settlement Workers in the Schools program of Citizenship and Immigration Canada).
- Ensure that services can respond to anticipated needs (e.g. post-traumatic stress, alcohol abuse).

- Increase the cultural competency of institutions to better respond to multicultural needs.

- Provide training for health professionals and others around gender and cultural sensitivity.

- Facilitate acceptance and incorporation of community-identified traditional healing.

- Provide communities with culturally appropriate avenues for coping

\section{Training, Supervision, and Monitoring}

Training and supervision should be carried out by mental health specialists, or under their guidance, for a substantial period of time in order to ensure lasting effects of training and care. Monitoring indicators should be determined before starting activities and should include resources (including available and pre-existing), processes, and outcomes.

- Base program and training initiatives on prior community needs assessments.

- Identify both immediate and long-term needs and incorporate both into training materials.

- Ensure that there is a collective decision-making body of experts and key stakeholders to develop guidelines and procedures for training and supervision.

- Involve organizations both within and outside of affected communities.

- Identify training resources within the relevant communities to build on existing expertise.

- Ensure "core readiness" by compiling in advance a list of relevant specialists and experts.

- Provide cultural competency training to researchers, policy makers, and service providers.

- Provide awareness training to community members about the mental health impact of acute emergencies and effective coping strategies.

- Use a "cascade" model of training, in which leaders are trained to train others.

- Maintain effective communication to facilitate information flow and collaboration.

- Develop indicators of training and programmatic success.

\section{Relationships with the Larger Society, Other Cultural Communities, and Transnational Ties}

These additional recommendations were made in recognition of the special situation of diaspora communities.

- Build better partnerships to reduce competition for resources among cultural communities.

- Keep in mind that mass media can be helpful, but caution is needed to ensure that representations of 
communities are accurate and that their best interests remain paramount.

- Encourage awareness of the fact that some newcomers, especially refugees, may be vulnerable due to prior trauma experience. However, many immigrants share similar settlement stresses.

- Recognize that attitudes about the homeland can differ among diaspora communities. These depend on many factors, including length of stay and sense of belonging in the new society.

- Involvement of foreign-trained health professionals in culturally competent health service delivery might be helpful in distress relief initiatives, but they may have left countries where there is also a great need for their services.

- Pursue and support collaboration with institutions in the homeland, as many communities have transnational linkages and resources. For example, culturally competent program manuals regarding disaster and/or distress relief or alternative therapies developed in the homeland may provide very helpful insights in Canada.

Participants also offered several recommendations as strategic research priorities. Above all, they recommended that research initiatives be responsive to community needs and that communities be directly and meaningfully involved in any research, service, and policy-making initiatives that concern them. To enable collaborative research, they suggested grounding research in the principles of community development and providing funding and guidelines to support academic/community partnerships. They also recommended increasing cultural competence by developing culturally appropriate assessment tools, undertaking social intervention research in the area of cultural competence and mental health, conducting more evaluationbased research of distress relief initiatives, and ensuring that research findings are translated into concrete products and services that can be used to address existing community needs. Directions included researching specific issues within both diaspora and homeland communities to provide comparative data, for example, researching mental illnesses (e.g. PTSD) in Canada and in the country of origin (e.g. Sri Lanka).

In sum, the key message distilled from the Local Distress Relief Network and the workshop experience is that providing effective distress relief must involve meaningful collaboration between mental health practitioners, social institutions, and diaspora communities. They provide cultural expertise and social resources for recovery and resilience where it often matters most, on the community level. The positive outcomes of the workshop described demon- strate that refugee communities can and do provide leadership and vision, and that the ideas and organizational lessons shared here hold promise for promoting refugee mental health in the diaspora.

\section{Notes}

1. The LDRN did not exclude Sinhalese Sri Lankans and Indonesians who were also affected by the tsunami, but these populations in Toronto are comparatively small, at 8,000 and less than 1,000 , respectively.

2. Morton Beiser, Laura Simich, and Nalini Pandalangat, "Community in Distress: Mental Health Needs and Help-seeking in the Tamil Community in Toronto," International Migration 41, no. 5 (2003): 233-245; Zachary Steel, Derrick Silove, Kevin Bird, Patraick McGorry, and P. Mohan, "Pathways from War Trauma to Posttraumatic Stress Symptoms among Tamil Asylum Seekers, Refugees and Immigrants," Journal of Traumatic Stress 12, no. 3 (1999): 421-435.

3. Steven Weine, Yael Danieli, Derrick Silove, Mark Van Ommeren, John A. Fairbank, and Jack Saul, "Guidelines for International Training in Mental Health and Psychosocial Interventions for Trauma Exposed Populations in Clinical and Community Settings," Psychiatry 65, no. 2 (2002): 156-164.

4. Mark Van Ommeren, Shekhar Saxena, and Benedetto Saraceno, "Mental and Social Health during and after Acute Emergencies: Emerging Consensus?" Bulletin of the World Health Organization 83, no. 1 (2005):75-76; Mitchell G. Weiss, Benedetto Saraceno, Shekhar Saxena, and Mark Van Ommeren, "Mental Health in the Aftermath of Disasters: Consensus and Controversy," Journal of Nervous and Mental Disease 191, no. 9 (2003): 611-615; World Health Organization, Mental Health in Emergencies: Mental and Social Aspects of Health of Population Exposed to Extreme Stressors (Geneva: WHO Department of Mental Health and Substance Dependence, 2003).

5. See pages 488-455 in Steven Vertovec, "Conceiving and Researching Transnationalism," Ethnic and Racial Studies 22, no. 2 (1999): 447-462.

6. Krishanthy Shu, A Report on Developing Grief Counselling and Mental Health Services (Toronto: Vasantham, A Tamil Seniors Wellness Centre, 2005).

7. Daya Somasundaram and Willem A.C.M. van de Put, "Management of Trauma in Special Populations after a Disaster," Journal of Clinical Psychiatry 67 (2006), Supplement 2: 64-73.

8. Fran Norris and Margarita Alegria, "Mental Health Care for Ethnic Minority Individuals and Communities in the Aftermath of Disasters and Mass Violence," CNS Spectrums 10, no. 2 (2005): 132-140.

9. Dinesh Bughra and Mark Van Ommeren, "Mental Health, Psychosocial Support and the Tsunami," International Review of Psychiatry 18, no. 3 (2006): 213-216.

10. The study was led by Dr. Morton Beiser (principle investigator). Research team members were Laura Simich, Anneke Rummens (co-investigators), Nalini Pandalangat and Abi- 
manyu Singam (project coordinators). Project partners included the Family Service Association of Toronto, the South Asian Women's Centre, the Society for the Aid of Ceylon Minorities (SACEM), and the Senior Tamils' Centre. The project Community Advisory Board also included representatives from Vasantham, A Tamil Seniors Wellness Centre; St. Joseph's Hospital Women's Health Centre; Mount Sinai Hospital, Ethnocultural ACT Team; Providence Centre, the Tamil Caregiver Project; and Community Resource Consultants of Toronto.

11. Beiser, Simich, and Pandalangat.

12. We thank the Institute for Health Services and Policy Research (IHSPR), Canadian Institutes for Health Research, for providing workshop funding and the Department of Psychiatry, University of Toronto, for in-kind support. We would also like to express gratitude to many members of the Toronto Tamil community who led the way.

13. Beiser, Simich, and Pandalangat.

14. Van Ommeren, Saxena, and Saraceno.

15. Derrick Silove, "The Best Immediate Therapy for Acute Stress Is Social," Bulletin of the World Health Organization 83, no. 1 (2005): 75-76.

16. Derek Summerfield, "What Exactly Is Emergency or Disaster 'Mental Health'?" Bulletin of the World Health Organization 83, no. 1 (2005): 76-77.

17. Silove, 75 .

18. Summerfield, 76 .

19. Ibid.

20. Weiss et al.

21. Ibid., 614.

22. Weine et al.

23. Maurice Eisenbruch, "From Post-traumatic Stress Disorder to Cultural Bereavement: Diagnosis of Southeast Asian Refugees," Social Science and Medicine 33, no. 6 (1991): 673-680.

24. Ibid., 674 .

25. Ibid., 678 .

26. Jack Saul (with Shquipe Ukshini, Afrim Blyta, and Shukrie Statovci), "Strengths-based Approaches to Trauma in the Aging," in Living Beyond Loss: Death in the Family, ed. F. Walsh and M. McGoldrick (New York: W.W. Norton, 2004).

27. Ibid., 300 .

28. Ibid.

29. Judith Landau and Jack Saul, "Facilitating Community Resilience in Response to Major Disaster," in Living Beyond Loss:
Death in the Family. ed. F. Walsh and M. McGoldrick, (New York: W.W. Norton, 2004).

30. Lamaro Patience Onyut, Frack Neuner, Elizabeth Schauer, Verena Ertl, Michael Odenwald, Maggie Schauer, and Thomas Elbert, "The Nakivale Camp Mental Health Project: Building Local Competency for Psychological Assistance to Traumatized Refugees," Intervention 2, no. 2 (2004): 90-107.

31. Lamaro Patience Onyut, Frack Neuner, E. Schauer, V. Ertl, M. Odenwald, Margaret Schauer, and Thomas Elbert, "Narrative Exposure Therapy as a Treatment for Child War Survivors with Posttraumatic Stress Disorder: Two Case Reports and a Pilot Study in an African Refugee Settlement," BMC Psychiatry 5, no. 7 (2005), <http://www.biomedcentral.com/ $147-24 \mathrm{X} / 5 / 7>$.

32. R. Balakumar, A. Barath, G. Chanda, A. Kulan, G. Namanan, and D. Ravindran, eds., Resilience and Resurgence: Rehabilitation and Reconstruction after the Tsunami Disaster in Sri Lanka (Toronto: Caldwell, 2005).

33. More information on these programs can be accessed at $<$ http://www.itspnyc.org > and <http://www.communityresilience.org/nyc $>$.

34. Van Ommeren et al.

Laura Simich, PhD, is assistant professor in the Departments of Psychiatry and Anthropology, University of Toronto, and scientist, Social Equity and Health Research, Centre for Addiction and Mental Health. Joanna Anneke Rummens, PhD, is health systems research scientist with the Community Health Systems Resource Group, Hospital for Sick Children, Toronto, and assistant professor of Culture, Community and Health Studies and Women's Mental Health, Department of Psychiatry, University of Toronto. Lisa Andermann, MD, FRCPC, is assistant professor, Culture Community and Health Studies, Department of Psychiatry, University of Toronto; and psychiatrist, Mount Sinai Hospital, Toronto. Ted Lo, MBBS, MRCPsych, FRCPC, is a psychiatrist with Culture, Community and Health Studies, Department of Psychiatry, University of Toronto and the General Psychiatry Program, Centre for Addiction and Mental Health. 